$10 \mid 2016$

Travail immatériel et immesurable ?

\title{
Antonio Negri. La necessità della svolta ontologica
}

Irene Viparelli

URL: http://journals.openedition.org/grm/888

DOI: $10.4000 / \mathrm{grm} .888$

ISSN: $1775-3902$

\section{Editore}

Groupe de Recherches Matérialistes

Notizia bibliografica digitale

Irene Viparelli, « Antonio Negri. La necessità della svolta ontologica », Cahiers du GRM [En ligne],

10 | 2016, mis en ligne le 23 décembre 2016, consulté le 24 avril 2019. URL : http://

journals.openedition.org/grm/888; DOI : 10.4000/grm.888

Questo documento è stato generato automaticamente il 24 aprile 2019.

(c) GRM - Association 


\title{
Antonio Negri. La necessità della svolta ontologica
}

\author{
Irene Viparelli
}

\section{NOTE DELL'AUTORE}

Lavoro svolto nell'ambito di un programma di post-dottorato, finanziato dalla "Fundação para a Ciência e Tecnologia".

\section{Introduzione}

1 Gli anni '80 rappresentano indubbiamente il momento di una profonda "rottura" nel dispositivo teorico di Negri; l'abbandono di Marx come referenza teorica esclusiva e l'ibridazione della teoria marxiana con la filosofia di Spinoza e, in seguito, con il poststrutturalismo francese. Insomma, come ammette lo stesso Negri in Pipe-line, riflettendo sulla sua adesione all'operaismo :

La ripresa del discorso filosofico corrisponderà per me al riaprirsi dell'irrequietezza vitale - e, perché non dirlo subito, ad alcune sconfitte politiche subite sul terreno costituito dai principi filosofici che ora venivo assumendo ${ }^{1}$.

2 Seguendo Negri, quindi, bisognerebbe interpretare la crisi dei "principi filosofici" che erano alla base dell'operaismo e il parallelo passaggio al punto di vista dell'ontologia costituente come effetti fondamentali della fine del periodo militante. A nostro avviso, però, questa lettura dello sviluppo teorico, se indubbiamente permette a Negri di mantenersi fedele al postulato operaista della costituzione pratica delle categorie teoriche, non sembra in realtà fornire una spiegazione esaustiva del percorso teorico di Negri. Tanto l'esigenza dell'allontanamento dalle categorie operaiste quanto quella di rifondare la teoria rivoluzionaria sul terreno ontologico, infatti, si trovano a nostro avviso già espresse negli scritti degli anni '70 e rappresentano il risultato fondamentale della radicalizzazione della critica delle categorie marxiane. 
Il '68, le lotte di liberazione nazionale, l'emergere di nuove soggettività antagoniste e la parallela marginalizzazione della classe operaia tradizionale avevano posto l'esigenza di una riformulazione delle problematiche che erano state fondamentali nel corso del decennio precedente. Da un lato, infatti, la strategia operaista, che opponeva il Marx "soggettivista" allo stalinismo e all'interpretazione dominante negli organi ufficiali del movimento operaio, si rivela insufficiente; dall'altro si afferma l'esigenza di una rivisitazione critica delle principali categorie marxiane e leniniane, capace di svelarne i limiti teorici e politici.

È la categoria dell'oltre che, acquisendo di fatto due significati differenti e complementari, esprime tale necessità di approfondimento della critica e di "superamento dell'operaismo". Prima di tutto, infatti, oltre indica un'opposizione interna al discorso marxiano : Grundrisse, oltre il Capitale; soggettivazione delle categorie dialettiche oltre ogni riduzionismo oggettivista ; operaismo oltre ogni idealismo del capitale. In secondo luogo, oltre esprime l'esigenza di "superare" gli stessi Grundrisse, la stessa dialettica soggettivista, spingendo la teoria oltre la prospettiva dello stesso Marx, verso una Neue Darstellung che, strutturata sul presupposto della valorizzazione autonoma del nuovo soggetto proletario, dell'operaio sociale, sappia rifondare la teoria rivoluzionaria su presupposti adeguati all'attuale fase della lotta di classe. Tale passaggio dall'ermeneutica alla creazione teorica pone l'esigenza del definitivo superamento del metodo operaista e della rifondazione ontologica della teoria rivoluzionaria ${ }^{2}$.

Nel corso del presente articolo, attraverso una rivisitazione dei testi di Negri degli anni '70, cercheremo di mostrare che la "svolta filosofica" degli anni '80, lungi dall'esser riducibile a mero "riflesso teorico" della "sconfitta militante", risponde piuttosto ad una necessità eminentemente teorica, risultante dall'approfondimento della critica di Marx e dalla concettualizzazione della nuova realtà dell'operaio sociale.

\section{Limiti metodologici}

6 La riflessione sui limiti della teoria marxiana si struttura a partire dal problema del "metodo" e, nello specifico, dal problema del "cominciamento": i Grundrisse rappresentano « il punto più alto dell'analisi e dell'immaginazione-volontà rivoluzionaria di Marx $»^{3}$, sono quindi "oltre" il Capitale, esattamente perché hanno un ordine di esposizione differente ; perché non cominciano con l'astratta teoria del valore, ma con la sezione "Denaro".

7 La fondazione della critica dell'economia politica sulla teoria del valore è, infatti, « un metodo più idealista, più hegeliano, malgrado ogni intenzione e dichiarazione in contrario $»^{4}$. $\mathrm{E}$, in altre parole, il segno inequivocabile di un "residuo di idealismo" nel discorso marxiano su cui si sono edificate, nel corso del XX secolo, tutte le interpretazioni "economiciste" e "idealiste", quindi, in ultima istanza, revisioniste della teoria di Marx.

8 L'immediata posizione della teoria del valore, secondo Negri, permette infatti di ipotizzare una sua presunta autonomia dalla teoria del plusvalore, supponendo quindi che sia storicamente possibile una realizzazione del valore senza sfruttamento. Una presunta "giusta" realizzazione della legge del valore che, strutturata sull'equilibrio tra produzione del valore e salario, identifica e confonde emancipazione comunista e pianificazione socialista ; generalizzazione sociale del lavoro salariato e sua soppressione.

Ben diverso è l'attacco dei Grundrisse : 
Il denaro è una cosa concreta che contiene tutti i dinamismi e le contraddizioni del valore, sia dal punto di vista formale che da quello sostanziale, senza possedere la vuota astrazione del discorso sul valore ${ }^{5}$.

Il denaro è l'antitesi della "vuota astrazione" della teoria del valore, poiché necessariamente presuppone l'esistenza concreta, storica, della società capitalistica sviluppata, in cui da un lato la classe operaia, persa la sua originaria fisionomia di "mero oggetto" dello sfruttamento, si è ormai affermata come soggetto antagonista; dall'altro, e conseguentemente, il capitale ha perso la sua struttura di "privata" e si è imposto come capitale sociale ${ }^{6}$.

11 Cominciare con il "Denaro" significa quindi rifiutare di dedurre il rapporto antagonistico di classe da una neutra relazione economica tra "persone astratte", che producono e scambiano la merce al "giusto" valore, per assumerlo come immediato presupposto dell'analisi. Significa, in altre parole, ricondurre lo sviluppo del capitale all'antagonismo di classe, in quanto rapporto dinamico in cui la tendenza proletaria all' « intensificazione

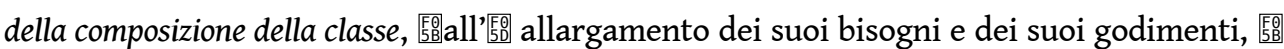
all'頤 innalzamento del valore del lavoro necessario alla sua riproduzione $»^{7}$ definisce la parallela tendenza del capitale alla sua progressiva socializzazione, alla sua trasformazione in soggettività politica.

Insomma, cominciare con il "Denaro", significa assumere la "prospettiva operaista" del primato delle forze produttive sui rapporti di produzione, fondando la critica dell'economia politica non sulla "legge del valore", ma la sua crisi, sull'essenziale precarietà del rapporto di classe.

\section{Dallo Stato-piano allo Stato crisi}

13 Si potrebbe paradossalmente dire che, mentre nel Capitale le categorie sono generalmente modellate sul capitale privato e concorrenziale, nei Grundrisse esse sono modellate su uno schema tendenziale di capitale sociale. ${ }^{8}$

La superiorità dei Grundrisse rispetto al Capitale è riconducibile, in ultima istanza, alla sua "forza anticipatrice", alla capacità di Marx di andare oltre i limiti del suo tempo per sviluppare l'analisi dal punto di vista della "crisi dello Stato piano"; ovvero dal punto di vista della crisi della forma assunta dal capitale tra il 1917 e l'inizio degli anni '70.

La Rivoluzione d'Ottobre rappresenta infatti l'origine di una nuova epoca capitalistica, strutturata su una logica di sviluppo assolutamente nuova e caratterizzata dall'affermazione storica della classe operaia come soggettività politica antagonista :

D'ora in poi la teoria dello stato dovrà fare i conti non solo con i problemi inerenti al meccanismo di socializzazione dello sfruttamento, ma con una classe operaia politicamente identificata, divenuta soggetto, - con una serie di movimenti materiali che già dentro la loro materialità portano intera la connotazione politica rivoluzionaria9.

16 L'affermazione dello Stato-piano, che associa pianificazione della produzione e politiche di redistribuzione della ricchezza, esprime la presa di "coscienza", da parte del capitale, della avvenuta metamorfosi storica: l'antagonismo è assunto come momento ineliminabile dello sviluppo del capitale e forza propulsiva dello sviluppo.

Il progetto keynesiano (e poi shumpeteriano) dell'"equilibrio dinamico" tra gli opposti interessi di classe, però, si deve rivelare intimamente e essenzialmente contraddittorio : 
La potenza capitalistica nell'assorbire le forze produttive è puramente storica, Marx direbbe "casuale", cioè non dotata di forza razionale, ma "irrazionale", laddove l'antagonismo che caratterizza la fondazione del rapporto è predisposto alla rottura, alla scissione, all'esplosione ${ }^{10}$. tentativi di integrarlo nel piano di sviluppo del capitale, trasformando le "misure riformiste" del capitale in armi rivoluzionarie ; l'"equilibrio" tra le classi in base per espandere permanentemente il movimento di autovalorizzazione di classe. Conseguentemente, la lotta di classe dell'operaio massa progressivamente sottrae il salario ad ogni "misura capitalistica", trasformandolo dapprima in "variabile indipendente", autonoma rispetto alla logica del profitto e dipendente appena dalla forza della soggettività operaia ${ }^{11}$, poi in "ostacolo radicale dello sviluppo ${ }^{12}$; in elemento di dissoluzione dei presupposti dello Stato-piano:

Il sogno riformista s'infrange sulla stag-flation e cioè sull'accumularsi di meccanismi di stagnazione (appiattimento del saggio del profitto) e delle spinte all'inflazione, spinte da salario, richieste di appropriazione del profitto lordo da parte della nuova massa di proletari riunificata in soggetto produttivo e - insieme, corrispondentemente potenzialmente sovversivo. ${ }^{13}$ frazione di lavoro necessario, perviene ad annullare completamente i margini del profitto, realizzando storicamente la legge della "caduta tendenziale del saggio di profitto", dissolvendo le condizioni della valorizzazione del capitale e, con loro, la funzione storica, la necessità essenziale, delle relazioni capitalistiche di produzione.

La dissoluzione dei presupposti riformistici su cui si era edificato lo Stato-piano impone, per Negri, una nuova, radicale, metamorfosi del capitale : ancora una volta, come già dopo il '29, si devono stabilire i nuovi presupposti della valorizzazione e così rifondare le condizioni di esistenza del capitale.

21 La prima e fondamentale condizione della persistenza storica del capitale in assenza delle condizioni oggettive della sua valorizzazione è la trasformazione del capitale in "comando":

Ora, quando la crisi storica dei rapporti di classe giunge al punto più profondo, la logica del comando deve cercare di esprimersi da sola. Produzione di merci a mezzo di comando : con ciò ogni rapporto tra valore e prezzo, fra produzione e circolazione viene meno 14 .

Quando l'assolutezza del "rifiuto proletario" annulla le condizioni storiche di ogni dialettica di capitale, il rapporto di classe si trasforma in una relazione eminentemente politica tra soggettività antagoniste, in rapporto di potere.

La dissoluzione delle condizioni economiche della valorizzazione e la trasformazione del capitale in "forza politica" rispondono all'esigenza di ripristinare una dinamica valorizzatrice, in una nuova forma, assolutamente "inedita", compiutamente "politica", che sappia garantire il persistere di una "orizzonte" del valore a partire dalla definitiva crisi della legge del valore. Attraverso l'amministrazione, il capitale estende il proprio controllo sulla totalità delle relazioni sociali e, portando a compimento il passaggio dalla sussunzione formale alla sussunzione reale ; sottomette l'intera sfera della riproduzione, la totalità delle relazioni sociali, alla logica del profitto :

È lo Stato della rendita, della rendita politica. Il valore assoluto cui tutti gli altri valori gerarchici debbono commisurarsi è il potere politico. Sulla base di questo valore assoluto si dà una scala di rendite differenziali, il cui valore è calcolato dalla maggiore 
o minore distanza dal centro, dal luogo di produzione del potere. (...) La collocazione gerarchica, la struttura corporativa, la collocazione dei corpi separati : tutto si articola su questa logica. Le rendite differenziali sono il segno variabile della variabilità dell'inserimento nella gerarchia, nell'articolazione del comando. Questa è dunque l'unica forma nella quale l'indifferenza può determinarsi. (...) Che cos'è lavoro produttivo nello Stato-rendita ? È, dal punto di vista del capitale, quella parte del lavoro sociale che viene sindacalizzata, corporativizzata, collocata nella separazione della gerarchia statale. L'indifferenza al valore prodotto è da questo punto di vista pari all'attenzione prestata ai coefficienti di fedeltà al sistema. Il mercato del lavoro - cioè la forza-lavoro complessiva nella sua relativa indipendenza - viene selezionato secondo i valori gerarchici che il sistema propone ${ }^{15}$. dell'astratto lavoro sociale, perde definitivamente ogni "misura oggettiva", ogni significato economico, ogni necessità storica, ogni funzione progressiva, configurandosi semplicemente come strumento di dominio, come potere di sottomissione delle forze produttive sociali. Il valore capitalistico diviene così una "illusione", una mera "immagine di profitto", una "pura mistificazione" prodotta mediante la logica del comando :

Ma è ben viva politicamente questa mistificazione ! Certo, perché solo in tal modo il capitale complessivo riesce a riproporre una regolo di dominio e di potere come "rapporto con se stesso", a porsi come "soggetto del circolo di profitto". Accettando la caduta del saggio di profitto, riattivando solo formalmente nella subordinazione della società alla fabbrica la legge del valore, il capitale controlla comunque e comanda tutte le valenze dell'unificazione dello sfruttamento e le fa valere come separate. Nella caduta di ogni motivazione della propria esistenza il capitale penetra comunque l'intera articolazione sociale del lavoro, facendo valere come comando una legge del valore, un immagine del profitto laddove il plusvalore si inclina. (...) L'unica forma di produrre merci che resta al capitale è quella di farlo secondo una vuota logica di permanenza del suo dominio ${ }^{16}$.

La trasformazione del capitale in "potere di mistificazione", forza di sottomissione della totalità delle relazioni sociali alla "logica del profitto, lungi dal riuscire a "liberare" il capitale dal rapporto antagonistico con il lavoro produttivo, trasformando ogni lavoro in lavoro produttivo, impone piuttosto la ricomposizione del soggetto politico antagonista su tale nuovo piano sociale - il passaggio dall'operaio-massa all'operaio-sociale -, estendendo la lotta di classe alla totalità delle relazioni sociali : la sottomissione della totalità delle relazioni sociali alla logica del profitto.

Insomma la trasformazione del capitale implica, necessariamente, la parallela metamorfosi dell'operaio massa e la costituzione di un nuovo soggetto politico che, venuta meno ogni necessità economica della dialettica di capitale e lavoro salariato, si caratterizza per la sua autonomia : "la forza produttiva divorzia dal capitale ${ }^{17}$, si rende "autonoma", rifiuta ogni mediazione con il capitale e, organizzando la produzione e riproduzione sociale indipendentemente, compie il passaggio «da forza-lavoro a forzainvenzione.

Definiamo la forza-invenzione come capacità della classe di nutrire nella più completa indipendenza antagonistica il processo di autovalorizzazione proletaria, di fondare quest'indipendenza innovativa sulla energia intellettuale, astratta come specifica forza produttiva (sempre più in maniera esclusiva) ${ }^{18}$.

Così l'autovalorizzazione di classe, l'“autonoma creatività" delle forze produttive sociali, obbligando il capitale ad un continuo inseguimento delle forze produttive sociali per sottometterle alla logica del comando, diventa un fattore immanente di destabilizzazione e destrutturalizzazione, che trasforma la sfera della riproduzione in "campo aperto - 
esattamente come il terreno della produzione - della lotta fra le due classi $»^{19}$, luogo di battaglia tra forze inconciliabili, spazio di una guerra civile permanente.

\section{Neue Darstellung. Oltre i Grundrisse}

La definitiva dissoluzione della funzione economica del capitale e la sua trasformazione in pura forza politica, in potere che si valorizza esclusivamente attraverso il comando, segnano il punto culminante dello sviluppo capitalistico e, parallelamente, dell'analisi marxiana. Inseguendo la tendenza antagonistica Marx infatti, nel celebre frammento sulle macchine, offre una vera e propria fenomenologia della società capitalistica "pervenuta al suo concetto"; una società in cui «il processo lavorativo è assunto come semplice elemento del processo di valorizzazione $»^{20} \mathrm{e}$, conseguentemente, la totalità le forze produttive socializzate, in primo luogo la scienza, sono ormai pienamente sussunte alla logica del comando capitalistico. Qui, all'apice dell'analisi critica, Marx arriva a cogliere la "dissoluzione capitalistica del valore d'uso operaio $"^{21}$, la generale sottomissione delle dinamiche di realizzazione dei bisogni alla logica del comando capitalistico, senza però riuscire a trarne, secondo Negri, le necessarie conseguenze teoriche e politiche, prima fra tutte quella della trasformazione di ogni lavoro in lavoro produttivo e. Marx, infatti, resta vincolato ad una definizione decisamente troppo riduttiva del concetto di lavoro produttivo,

legata all'assiologia socialista del lavoro manuale. Condizionata da questa, anche quando tutte le condizioni teoriche erano mutate. E quanto profondamente mutate ! Perché infatti era solo sulla base di una dislocazione completa del concetto di lavoro produttivo che ora la stessa definizione di classe rivoluzionaria poteva essere data. Trattenersi sui livelli dell'assiologia socialista nella definizione di questo concetto, quando tutto l'apparato sistematico e definitorio era stato dislocato in avanti, era francamente inutile e infruttuoso. Marx subisce qui, dannosamente, il limite di organizzazione del movimento operaio ${ }^{22}$.

Insomma, secondo Negri, «il ritardo dell'organizzazione operaia (...) blocca l'avanzamento teorico ${ }^{23}$, impedendo a Marx di compiere quell'ulteriore dislocazione dell'analisi dall'oggettivo al soggettivo, che sola gli avrebbe permesso di anticipare teoricamente, a partire dalla fenomenologia della società del capitale giunto a maturazione, la nuova fisionomia della classe rivoluzionaria e della lotta di classe.

Tale limite del discorso marxiano determina un essenziale squilibrio dell'analisi : da un lato Marx, pienamente cosciente della trasformazione del capitale circolante in capitale "produttivo nella forma della pianificazione e del controllo della riproduzione della società $\|^{24}$, riesce a cogliere la tendenza alla progressiva approssimazione, fino all'identificazione, dei concetti di produzione e riproduzione. Dall'altro, però, l'inadeguatezza del concetto di lavoro produttivo gli impedisce di dedurne la necessaria costituzione di una nuova figura proletaria, quella dell'"operaio sociale", e l'estensione dell'« antagonismo di classe su tutto il terreno della società $»^{25}$, su tutta la sfera della riproduzione.

31 Il frammento sulle macchine, punto più alto dell'analisi, si rivela così esser anche il luogo teorico in cui diventano evidenti i "limites assoluti", le deficienze fondamentali della teoria di Marx ; in cui quindi emerge la necessità di un'ulteriore dislocamento dell'analisi oltre Marx : non solo oltre il Capitale ma oltre gli stessi Grundrisse. Lo squilibrio tra premesse teoriche e conclusioni, in altre parole, definisce il terreno su cui inaugurare un processo 
teorico assolutamente creativo, che riesca ad adeguare la teoria rivoluzionaria ad una contemporaneità che, in virtù dell'universalizzazione e assolutizzazione della logica antagonistica, appare ormai definitivamente oltre la prospettiva teorica di Marx :

Oggi è dunque possibile andare "oltre Marx", su questa strada, le cui premesse Marx ha decisamente proposto. Ma il salto in avanti, la figura determinata di realizzazione del comunismo, la sua dinamica sono elementi talmente fortemente connotati che davvero val qui la pena di ripeterci, increduli : siamo andati oltre $\operatorname{Marx}^{26}$.

Marx ha radicalmente trasformato la dialettica e, liberandola dall'idealismo, l'ha saputa rifondare sul presupposto materialista dell'antagonismo, riuscendo così a sviluppare l'essenziale tendenza del capitale fino a coglierne la forma "compiuta", il suo concetto pienamente sviluppato. Su tale terreno, l'ulteriore sviluppo della teoria rivoluzionaria deve necessariamente configurarsi, per Negri, come una seconda "rivoluzione metodologica" che sappia abbandonare definitivamente i presupposti dialettici dell'analisi : quando il capitale si è ormai trasformato in pura potere di mistificazione e le nuove forze produttive, immediatamente sociali, si sono rese autonome, i presupposti su cui si fondava la dialettica della separatezza sono ormai definitivamente dissolti.

Il nuovo metodo, conseguentemente,

smette di svolgersi su un ritmo binario, smette anche di accettare la realtà fantasmatica dell'avversario sul suo orizzonte. Cancella la dialettica anche solo come orizzonte. Rifugge ogni formula binaria. Qui il processo antagonistico tende all'egemonia, alla distruzione ed all'annullamento dell'avversario. Negare la dialettica, questa formula eterna del pensiero giudaico-cristiano, questa perifrasi per dire nel mondo occidentale - razionalità : in Marx noi abbiamo letto il più avanzato progetto verso questa distruzione, abbiamo visto svolgersi i primi giganteschi passi in questa direzione. Dobbiamo ora procedere a fondo. Solo su questo terreno, allora, potremo cominciare a parlare di nuove categorie, non del capitale ma del rovesciamento del capitale ${ }^{27}$.

Da un lato "la dialettica è restituita al capitale ${ }^{28}$, configurandosi come la "mediazione" mistificatrice che fonda la nuova forma "politica" della "valorizzazione capitalistica". Il materialismo, parallelamente, "diviene l'orizzonte esclusivo, innervato dalla sua logica antagonistica e soggettiva $»^{29}$, l'autoesposizione della potenza costitutiva, inventiva, delle nuove forze produttive autonome, il disvelamento della radicalità del nuovo antagonismo, strutturato sul presupposto dell'impossibilità di ogni relazione con il nemico di classe.

Il metodo di Marx si era mosso dalla demistificazione all'inversione delle categorie dell'economia politica classica, dallo smascheramento della falsità del punto di vista borghese, che pone la mediazione capitalistica come motore dello sviluppo, alla fondazione della storicità al capitalismo sulla discontinuità, sulla separazione e sull'antagonismo proletario. Il nuovo metodo, rappresentando la definitiva autonomizzazione delle forze produttive sociali dal capitale, dovrà compiere un ulteriore passaggio "dall'inversione alla costituzione $"^{30}$, configurandosi come una Neue Darstellung che, abbandonando il presupposto dialettico del vincolo capitale-classe operaia, riesca a esprimere la potenza immanente nella autonoma "forza-invenzione" dell'operaio sociale :

Con la tematica della costituzione noi entriamo in una fase teorica in cui la concreta determinazione dei comportamenti proletari, la prassi collettiva del proletariato divengono un motore teorico, un tessuto di proposizione teorica, un soggetto che ha una straordinaria libertà e forza di autoesposizione. (...) La Neue Darstellung, nel 
processo maturo della costituzione dell'individuo collettivo del comunismo, deve

sempre più divenire una Selbst-Darstellung ${ }^{31}$.

Il nuovo modo di esposizione è "comunismo nella forma della transizione $»^{32}$ : la potenza comunista immanente alla nuova pratica sociale dell'antagonismo, infatti, definisce la nuova e inedita tendenzialità storica che, dalla destrutturazione del capitale porta al processo costitutivo di una nuova realtà; "dall'autovalorizzazione all'autodeterminazione, all'indipendenza sempre più alta e completa del soggetto proletario, alla multilateralità dei suoi percorsi $»^{33}$.

\section{Conclusioni}

Il passaggio dalla Darstellung marxiana alla Neue Darstellung impone l'abbandono dei presupposti dialettici del marxismo e, con essi, l'allontanamento dal metodo operaista.

Il primato delle forze produttive sui rapporti di produzione, nella prospettiva operaista, costituisce un postulato esclusivamente astratto, nella misura in cui la sua realizzazione storica risulta esser sempre vincolata alla definizione del vincolo dialettico tra forze produttive e rapporti capitalistici di produzione. La categoria dell'antagonismo, conseguentemente, rappresenta la chiave metodologica per la comprensione dello sviluppo storico.

39 La concettualizzazione dell'operaio sociale, dislocando il postulato del primato delle forze produttive sul terreno concreto della storia, dissolve tale schema operaista e definisce i presupposti per una radicale "rivoluzione metodologica": da un lato il concetto di "autonomia" diventa il presupposto dell'analisi ; dall'altro, parallelamente, l'antagonismo perde il suo primato metodologico, per trasformarsi in risultato, effetto, dell'azione a posteriori del capitale sulle forze produttive autonome. È quest'ultimo infatti che, per garantire il persistere dell'orizzonte del valore nella crisi della legge del valore, sottomette le forze produttive sociali alla logica del profitto, dissolvendone l'autonomia e instaurando una dinamica antagonistica sull'intero arco della riproduzione sociale.

Tale nuovo primato metodologico della categoria di "autonomia", opponendo il piano della valorizzazione al piano della dialettica storica, pone l'esigenza teorica della rifondazione ontologica della teoria rivoluzionaria. Da un lato, le relazioni capitalistiche di produzione appaiono ormai come mero dominio di un potere politico-ideologico che, incapace di produrre sviluppo e tendenzialità, sopravvive bloccando i processi storicocreativi della "forza-invenzione". Dall'altro, parallelamente, la produzione, dissolta ogni dialettica con il capitale, si pone su un terreno assolutamente discontinuo, oltre i limiti di Marx, oltre i rapporti di produzione capitalistici, oltre il piano ontico.

Secondo me dunque siamo, appunto in questo periodo '73 - '77, in una situazione molto felice dal punto di vista teorico, felice nella misura in cui riusciamo appunto a determinare concretamente un terreno di sintesi tra molteplicità dei comportamenti sociali che vengono esprimendosi (e delle posizioni sociali che man mano si soggettivizzano) e quella che, dall'altro lato, è l'ipotesi teorica dell'operaio sociale, Salvo che, appunto, la stessa qualità della sintesi implica una modificazione. Non so, ma probabilmente - ed è una cosa che devo dire anche rispetto ai miei scritti - si è terribilmente indietro rispetto a questo salto qualitativo, a questa situazione teorica. Personalmente sento questi limiti in maniera molto forte. Devo fare autocritica in proposito: ho l'impressione di avere fino a questo momento funzionato più come colui che recupera una serie di testi marxiani, e li stende, li tira fino a coprire nuovi fenomeni ; mentre invece oggi il problema non è questo, 
ma quello di partire appunto da questi nuovi fenomeni e riverificare la tematica marxista alla loro luce, nella più completa indipendenza, a fronte dell'ortodossia, anche di quella corretta, del progetto teorico e politico, nella loro originalità ${ }^{34}$.

41 La "svolta filosofica" degli anni '80 si presenta allora come il risultato dell'insorgenza del problema del "ritardo teorico" degli scritti militanti degli anni '70 ; come il tentativo di rispondere alla sfida, posta - ma non risolta - dall'operaio sociale, rifondare la teoria rivoluzionaria oltre la dialettica, sul principio ontologico-costituente dell'autonomia.

A partire da qui, dalla individuazione della necessità genetica dell'ontologia costituente, si afferma un nuovo problema teorico : gli scritti della prima metà degli anni '80 riescono a rompere definitivamente con la dialettica e con l'operaismo? Sono all'altezza del compito che si propongono?

\section{NOTE}

1. Antonio Negri, Pipe-line. Lettere da Rebibbia, Roma, DeriveApprodi, 2009, p. 55.

2. In un certo senso, quindi, Tronti ha ragione quando afferma che l'esperienza operaista si conclude con la "morte di "classe operaia" " (Mario Tronti, Noi operaisti, Roma, DeriveApprodi, 2009 , p. 5). I presupposti teorici dell'“operaismo degli anni '70" sono infatti molto differenti rispetto alle premesse teoriche su cui si struttura l'operaismo.

3. A. Negri, Marx oltre Marx, Milano, Feltrinelli, 1979, p. 21.

4. Ibid., p. 50.

5. Ibid.

6. In tal modo, nella dialettica della separazione, « il risultato deve fungere altresì da presupposto. Il risultato (crisi e dissoluzione a livello del mercato mondiale) deve fungere altresì da presupposto (antagonismo e lotta a livello del rapporto di produzione)»(Ibid., p. 73).

7. Ibid., p. 83.

8. Ibid., p. 38 .

9. A. Negri, J. M. Keynes e la teoria capitalistica dello stato nel '29, in AA. VV, Operai e Stato. Lotte operaie e riforma dello stato capitalistico tra rivoluzione d'Ottobre e New Deal, Milano, Feltrinelli, 1973, p. 70.

10. A. Negri, Marx oltre Marx, cit., p. 82.

11. "Questa argomentazione, come molte altre elaborate dall'operaismo, aveva poco in comune con i precetti marxisti convenzionali. D'altra parte, anche se la nozione di Negri del lavoro come variabile indipendente nel rapporto di classe contraddiceva palesemente la lettera del primo libro del Capitale, poteva però trovare sostegno nel Volume III dell'opus magnum di Marx. Più importante della conferma dei sacri testi, comunque, era la testimonianza eloquente dei crescenti problemi dell'economia italiana con la produttività e il profitto » (Wright, (2002), 2008 p. 204).

12. A. Negri, I libri del rogo, Roma, Castelvecchi, 1997, p. 88.

13. Ibid., p. 89.

14. Ibid., p. 94.

15. Ibid., p. 272.

16. Ibid., pp. 95-96.

17. Ibid., p. 289.

18. Ibid., p. 292. 
19. Ibid., p. 221.

20. A. Negri, Marx oltre Marx, cit., p. 150.

21. Ibid., p. 151.

22. Ibid., p. 190.

23. Ibid., p. 189.

24. Ibid., p. 151.

25. A. Negri, I libri del rogo, cit., p. 221.

26. A. Negri, Marx oltre Marx, cit., p. 194.

27. Ibid., p. 197.

28. Ibid., p. 177.

29. Ibid., p. 177.

30. Ibid., p. 188.

31. Ibid., p. 189.

32. Ibid., p. 162.

33. Ibid., p. 177.

34. A. Negri, Dall'operaio massa all'operaio sociale. Intervista sull'operaismo, Verona, Ombre Corte, 2007, p. 127.

\section{RIASSUNTI}

Il presente articolo si propone di mostrare che la "svolta filosofica" di Negri, agli inizi degli anni '80, lungi dal rappresentare un momento di "discontinuità teorica", risulta piuttosto esser una risposta alla sfida teorica, posta dall'operaio sociale, di una radicale rifondazione della teoria rivoluzionaria sul nuovo presupposto dell'ontologia costituente. Il principio dell'autonomia delle forze produttive sociali, infatti, nei testi degli anni '70, rappresenta la base di una radicale "rivoluzione metodologica" che da un lato impone l'allontanamento dal metodo dell'antagonismo, quindi dall'operaismo ; dall'altro implica la costituzione di un nuovo quadro teorico, strutturato sulla separazione tra il piano ontico delle relazioni capitaliste e il terreno ontologico-costituente dell'autonoma produzione di valore.

Au cours de cet article, au travers d'une analyse des textes de Negri des années 70, on cherchera à montrer que le «tournant philosophique» des années 80 , loin d'être réductible au simple "réflexe théorique» de l' «échec militant», répond plutôt à une nécessité éminemment théorique, résultant de l'approfondissement de la critique de Marx et de la conceptualisation de la nouvelle réalité de l'ouvrier social.

\section{INDICE}

Indice geografico : Italie

Temi : ontologie

Mots-clés : Negri Antonio, ouvrier social, opéraïsme

indice cronologico années 1970, années 1980 


\section{AUTORE}

IRENE VIPARELLI

Irene Viparelli est docteur en philosophie, membre du CICP (Centro de Investigação em Ciência Política - Universidade de Évora - Portugal) et du IFP (Instituto de Filosofia Prática -

Universidade de Évora - Portugal). Actuellement elle est en train de développer un projet de post-doctorat portant sur Negri e Althusser. 